\title{
„Wir verteilen konsequent auf die Krankenhäuser der Region“
}

\section{Interview mit Hans-Georg Jung}

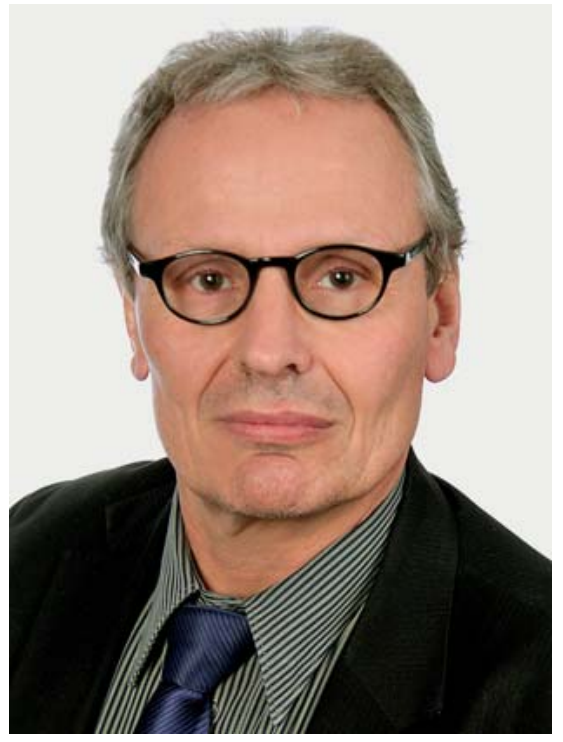

Der Leiter der „Medizinischen Gefahrenabwehr“ beim Gesundheitsamt Frankfurt am Main erklärt, wie Rettungsdienste mit einem neuen Onlinesystem Verletzte schneller und gezielter in die richtige Klinik bekommen auch und gerade nach möglichen Terroranschlägen.

Was ist Ihre Rolle hier im Gesundheitsamt der Stadt Frankfurt zum Thema Terror?

Wir erarbeiten hier grundlegende Konzepte für Vorbeugung und vor allem die Rettung nach solchen Katastrophen. Die konkrete Versorgung im Notfall leisten natürlich andere Akteure. Das ist dann Aufgabe der Leitstellen, der Rettungsdienste und Kliniken.

Sind wir vorbereitet für den Tag $\mathrm{X}$, den alle verhindern möchten, an dem es womöglich aber doch einen großen Terroranschlag gibt?
Für uns hier in Frankfurt würde ich sagen, dass wir in der Abstimmung der Akteure untereinander, zwischen Leitstelle, Rettungsdiensten und Kliniken heute gut vorbereitet sind.

Ein Defizit sehe ich nach wie vor hinsichtlich der qualifizierten Versorgung.

\section{Was meinen Sie da?}

Wir arbeiten seit Jahren auch mit Experten aus dem Traumanetzwerk der DGU zusammen und dort hat man schon länger darauf hingewiesen, dass viele Ärzte und Rettungskräfte ein massives Defizit beim Thema Versorgung von Schuss- und Explosionsverletzungen haben. Und lange Jahre, da nehme ich auch uns als Stadt Frankfurt nicht aus, wollte das keiner hören.

\section{Warum?}

Weil es sich um Verletzungen handeln würde, das war so ein Tenor, die am ehesten aus Kriegen bekannt sind. Die Bundeswehr hat aufgrund ihrer Arbeit in Spannungsgebieten seit 10 , seit 15 Jahren mit diesen Verletzungsmustern in der Tat zu tun. Deswegen besteht da natürlich eine Expertise, die wir nützen müssen, um unsere Leute auch für Rettungseinsätze und im Krankenhaus zu schulen.

Doch leider neigen wir in Deutschland zur Polarisierung. Anstatt solche Themen sachlich zu beurteilen und die Erfahrung abzufragen, die bei Ärzten im Sanitätsdienst da ist, haben viele Ärzte hierzulande gesagt, die spinnen, diese Unfallchirurgen, die wollen den Krieg vorbereiten. Wir machen Individualmedizin, wir machen keine Kriegsmedizin. Das ist allerdings nach meinem Eindruck vorbei, das Thema ist jetzt in den Köpfen, vielerorts wird geübt und gelernt.
Was ist zu lernen?

Die Basics dabei sind relativ leicht. Da geht es um eine Triage, da geht es um sofortige Blutstillung, insbesondere um Versorgung mit Tourniquets. Und um ein Airwegsmanagement. Details führen jetzt sicher zu weit. Unsere Rettungsdienste bis hin zu den leitenden Ärzten wurden bald nach den Attentaten in Paris vom November 2015 alle im Umgang mit Schuss- und Sprengverletzungen geschult.

Woher wissen Ihre Leute, wer was im Falle eines Falles zu tun hat?

Da sind grundlegende Dinge durch das Land Hessen in einem MANV-Rahmenkon-

\section{ZUR PERSON}

Hans-Georg Jung (Jahrgang 1959) leitet die Stabsstelle medizinische Gefahrenabwehr beim Gesundheitsamt der Stadt Frankfurt a. M. Der gelernte Rettungsassistent ist seit 1994 beim Gesundheitsamt der Stadt. Zunächst als Sachgebietsleiter für den Rettungsdienst bei der Berufsfeuerwehr zuständig, wechselte er 2004 zur Abteilung für regionale Krankenhausplanung. Dem Thema präklinische Versorgung und Koordination des Rettungswesens blieb er dennoch treu: 2010 ging in Hessen eine von Jung mitentwickelte Software an den Start: IVENA - der webbasierte interdisziplinäre Versorgungsnachweis eHealth. Das Programm ermöglicht Rettungsdiensten und Kliniken im Notfall den Austausch aller Daten über die Allokation der Patienten. Es ist ein gefragtes Tool, mittlerweile auch in anderen Bundesländern. 
zept und einem Katastrophenschutzkonzept festgelegt. Für Frankfurt haben wir vom Gesundheitsamt zusammen mit der örtlichen Feuerwehr einen KrankenhausEinsatzplan für Gefahrenlagen geschrieben.

\section{Steht er im Internet?}

Nein, wir publizieren ihn nicht, weil wir das nicht für zielführend halten. Es muss gar nicht jeder jedes Detail wissen. Sie können so einen Plan in seiner Gesamtheit ohnehin nicht kommunizieren. Auf diesen Plan beziehen sich vielmehr die Einzelanweisungen für den Rettungsdienst, für die leitenden Notärzte, für die Obersten Leiter im Rettungsdienst, OLRD, für die Krankenhäuser, und so weiter.

Was passiert als erstes, wenn es in Frankfurt einmal krachen sollte?

Wir haben in Frankfurt eine integrierte Leitstelle, in der Rettungsdienste und Feuerwehr arbeiten. Sobald sich, etwa schon aus der Notrufmeldung, in der Leitstelle Anzeichen für einen möglichen Terroranschlag ergeben, wird das sofort mit der Polizei kommuniziert. Und die wird sagen, stopp - zunächst keine Rettungsaktivitäten, wir checken den Tatort. Sie muss den Tatort erst freigeben.

Sobald er hindarf, wird der Rettungsdienst sofort eine erste Einschätzung der Lage an die Rettungsstelle geben, er wird die Größenordnung taxieren, wie viele Verletzte zu erwarten sind. Ob es sich um einen MANV50, einen MANV100, MANV250 handelt. Entsprechend der Anzahl der Verletzten ist bei uns genau festgelegt, wie viele Rettungsassistenten dann mit der Triage beginnen.

\section{Machen das nicht die Notärzte?}

Nein, die Triage ist bei uns in Frankfurt Aufgabe für die Rettungsassistenten und die Notfallsanitäter. Sie legen die Karten neben die Opfer.

Ist es richtig, dass es dabei 4 Karten verschiedener Farbe gibt? Grün für höchstens leicht verletzte Opfer, über gelb und rot für steigende Verletzungsgrade bis hin zu blau - ein Mensch, der so stark verletzt ist, dass er zumindest bei mangelnden Ressourcen nicht mehr behandelt wird. Ist das richtig?
Prinzipiell ja, aber blaue Karten verwenden wir nicht. Wir transportieren jeden, das ist unser Konzept.

Immer? Manche Ärzte und Leitlinien betonen gerade, dass bei einem MANV nach Terror eine Konzentration von Ressourcen nötig sein könnte, letztendlich auch mit der Konsequenz, dass Opfer, denen man keine Überlebenschance mehr gibt, nicht mehr behandelt werden.

Solch eine Entscheidung würde bei uns in Frankfurt ausschließlich das Krankenhaus treffen, nicht der Rettungsdienst. Wir gehen bei Terror auch gar nicht davon aus, in eine Lage zu kommen, wo wir das nicht durchziehen können. Wir haben uns die Zahlen aller Terroranschläge der letzten Jahrzehnte angeschaut. Weltweit lag die maximale Anzahl an Verletzten bei Terroranschlägen bislang maximal bei 300 bis 400 .

Also im schlimmsten Fall würden Sie einen MANV500 erwarten, 500 Verletzte als Maximum?

Ja, und das ist eine Dimension, von der ich behaupte, dass wir in Frankfurt in der Lage wären, jeden medizinisch zu versorgen.

Den Einsatz einer Karte blau halte ich nur für denkbar, wenn großflächig unsere Infrastruktur zusammenbricht. Das ist unwahrscheinlich.

Welche Versorgung findet am Tatort statt.

Es heißt bei uns „Treatment and Run“. Nur eine ganz kurze Erstversorgung, und dann sofortiger Transport in die Klinik. Nur das allernötigste, unsere ersten Leute am Tatort legen vielleicht einen Tourniquet an, schnelle Basics. Dann kommen weitere Rettungsassistenten und transportieren alle Opfer ab, zunächst die rot markierten, dann die Gelben, als letztes die Leichtverletzten. Die Leitung vor Ort haben der Oberste Leiter Rettungsdienst, der OLRD, und der Leitende Notarzt.

\section{Wohin kommen die Opfer?}

Dafür haben alle Akteure ja unser Programm IVENA.

\section{Was ist das?}

IVENA steht für interdisziplinärer Versorgungsnachweis für den Rettungsdienst. Es ist eine Software, die wir hier vom Ge- sundheitsamt zusammen mit einer Firma entwickelt haben. Mittlerweile ist es landesweit eingeführt, heute haben in Hessen fast alle Krankenhäuser und Leitstellen dieses Tool und auch schon die ersten Rettungsdienstfahrzeuge. Das Programm wird gehostet von einem Hochsicherheitszentrum des Landes Hessen, mit einer hohen Verfügbarkeit von 99,9 Prozent.

\section{Und was leistet es jetzt?}

IVENA regelt online die Kommunikation zwischen Leitstelle und Krankenhäusern. Im Programm sind die Kapazitäten der Kliniken für die Versorgung von Verletzten in der Stadt erfasst, sodass der Disponent in einer Leitstelle wie der OLRD genau nach diesen Kapazitäten die Zuteilung von Verletzten an die Häuser steuern kann. Jedes Haus ist mit Sollzahlen im Programm unterlegt, wie viele Patienten ihm bei welcher Gesamtzahl an Verletzten zugewiesen wird.

Wir nützen IVENA bei uns bereits in der Regelversorgung. Noch gibt der Rettungsdienst vor Ort die Daten an die Leitstelle per Funk durch, die sie dann in IVENA eingibt. Aber das stellen wir jetzt gerade ebenfalls auf Online um, der Rettungsdienst soll angeschlossen werden.

\section{Und die Kliniken?}

Bei einem Geschehen mit vielen Verletzten gibt es ein eigenes MANV-Zuweisungstool, das die Leitstelle aufruft und über das alle Krankenhäuser, die in der Nähe eines solchen Tatorts liegen, einen Voralarm erhalten, damit sie sich vorbereiten können.

Sobald der MANV genauer klassifiziert ist, löst die Leitstelle den konkreten Alarm aus. Und jetzt sieht jedes Krankenhaus sofort, wie viele Patienten es innerhalb eines gewissen zeitlichen Korridors bekommen soll. Für jeden zugewiesenen Patienten hat der OLRD dann auch schon eine Erstdiagnose eingespielt. Zugleich geben die Krankenhäuser ihre Daten ein, sie leisten zum Beispiel eine eigene Triage. Sollte sich dabei zum Beispiel zeigen, dass der Notdienst vor Ort etwa einen Patienten vorschnell in die Kategorie rot gestuft hat, wird das Haus diese Angaben nach gelb zurückstufen. Und schon weiß der Dienst vor Ort, dass er einen Platz weniger für „gelb“, aber noch einen Platz für „rot“ frei hat. 
Sind die Daten aus dem Traumanetzwerk der DGU auch im System hinterlegt?

Natürlich. Und sicherlich wird ein OLRD auf die Qualifikationen der Kliniken schauen, checken, welches Haus, das noch Kapazitäten frei hat, einen Patienten mit Polytrauma einschließlich Schädel-Hirn-Trauma fachlich am besten versorgen kann. Aber es sind alle Kliniken im System genau erfasst, denn wir brauchen bei hohen Opferzahlen womöglich alle Häuser der Region.

\section{Oder gar des Bundeslandes?}

Im Extremfall ja. Dafür können ja auch alle in das System hineinschauen, auch nicht betroffene Häuser weiter weg. Wenn es einen MANV hier in Frankfurt gäbe, könnten alle Landkreise und Kliniken quasi live die Verteilung der Patienten bei uns hier in Frankfurt mitverfolgen - auch wenn sie nicht mit in die Versorgung eingebunden sind. Diese Transparenz ist wichtig, gerade für den Fall, wenn ein Ereignis mal eskaliert. Wir hatten hier und da durchaus auch Widerstände, manche Häuser wollten ihre Daten nicht einspeisen. Aber nur mit dieser Transparenz kann das System auch seinen Zweck erfüllen. Wenn wir weitere Häuser brauchen, kann die Leitstelle über IVENA sofort auch entfernter liegende Kliniken einbinden.

Transportieren Sie die Opfer grundsätzlich zunächst in Kliniken, die am nächsten liegen?

Nein, wir würden die Patienten nach Möglichkeit genauso in der Region Frankfurt verteilen, wie es unser Plan vorsieht. Alles andere macht auch keinen Sinn, denn das würde ja nur Verkehrswege und Krankenhäuser verstopfen. Sie können auch nicht innerhalb von einer halben Stunde 10 rot eingestufte schwerverletzte Patienten in eine kleine Klinik bringen, nur weil die neben dem Tatort liegt, die Klinik würde Ihnen kollabieren. Die Patienten verteilen wir.

Einige Experten meinen, solch eine Verteilung sei im Chaos der akuten Situation nur schwer zu steuern.

Es kann Situationen geben, wo man tatsächlich in der Not viele, zu viele Patienten, in einem Haus unterbringen muss zumindest für eine bestmögliche Erstversorgung. Aber wir werden primär immer versuchen, die Leute genau nach den Ka- pazitäten, die uns IVENA zeigt, in der Fläche zu verteilen.

Gibt es Programme wie IVENA heute in allen Bundesländern?

Nein. Unser Programm stößt aber auf Interesse. Brandenburg hat IVENA bereits eingeführt, Berlin und Niedersachsen führen es gerade flächendeckend ein. Es gibt Anfragen und Interesse aus weiteren Regionen. In Bayern haben wir derzeit den Großraum München, Nürnberg und Regensburg. Auch NRW ist für uns schwierig, wir kennen auch dort viele Kliniken, die würden es gerne haben, aber NRW möchte wohl etwas Eigenes entwickeln. Rheinland-Pfalz hat ein eigenes Online-Tool.

Selbst manch andere Großstädte setzen offenbar noch auf Fax und Telefon bei einem MANV.

Das möchte ich nicht kommentieren, außer mit dem Hinweis, dass diese Prozesse im Zweifel möglich schnell gehen müssen. Und unser System für die Allokation der Patienten ist rasend schnell.

\section{Wer verdient an dem Programm?}

Die Leitstellen und Krankenhäuser müssen die Nutzungsrechte bei der von uns für den Vertrieb beauftragten Firma erwerben. Die Urheberrechte hält die Stadt Frankfurt zusammen mit der Firma. Dabei haben wir Spielregeln für die Preisgestaltung vereinbart. Unser Ziel ist, dass die Software überall dort zum Einsatz kommen kann, wo sie gewünscht wird. Ich verdiene nichts daran.

Wird mit IVENA in Frankfurt auch geübt? So ist es. Hessen ist das einzige Bundesland, das verpflichtend für alle Krankenhäuser alle 2 Jahre eine große Übung vorgeschrieben hat. Bei diesen Vollübungen erhält jedes Krankenhaus zum Teil auch unangekündigt je nach Kapazität zwischen 30, 50 oder 80 Patienten über IVENA zugewiesen. Wir üben dabei für jedes Haus die Sequenz an Patienten, die es bei einem MANV500 zu erwarten hätte. Wir haben für die Verteilung der Patienten eine Grundregel - 10 Prozent seiner Gesamtversorgungskapazität kann ein Krankenhaus für die Versorgung von Verletzten bei einem MANV leisten. Eine Uniklinik mit 800 Betten würde 80 Verletzte aufnehmen müssen.
Und muss damit quasi immer 80 Betten frei haben?

Nein, das Haus muss im Extremfall in der Lage sein, 80 Betten schnell frei zu bekommen. Wir haben vor allem bei Kontakten zu Kollegen in Israel gelernt, dass ein Umsteuern von Regelbetrieb zur Versorgung eines MANV im Krankenhausbereich möglich ist. Die Israelis können das. Wenn Krankenhäuser hierzulande sagen, sie können das nicht, dann stimmt das nicht. Man muss es nur üben.

Heißt, eine Uniklinik müsste im Fall des Falles 80 schwerverletzte Terroropfer versorgen?

Nein, es sind 80 Menschen mit unterschiedlich schweren Verletzungen. Wir erwarten, dass nach einem Terroranschlag 20 bis 25 Prozent der Patienten schwer verletzt sind. Weitere 30 Prozent fallen in die Kategorie gelb, 50 bis 60 Prozent in die Kategorie grün. Wir wissen, dass nach einem Attentat das Risiko groß ist, dass bei solch einem Ereignis gerade die vielen Leichtverletzten als Selbsteinweiser in die Kliniken kommen und dort die Hilfskapazitäten blockieren können, die in den ersten Stunden für die Versorgung der Schwerverletzten nötig sind. Auch den Umgang damit üben wir durch Zuweisung solcher Selbsteinweiser.

\section{Was tun?}

Zum einen sind wir schon vorher aktiv. Bei jedem größeren Ereignis, auf jeden Fall ab einem MANV250 haben wir in der Stadt 2 sogenannte Leichtverletztensammelstellen, zu denen wir diese Betroffenen mit Bussen hinbringen und ihnen ein niederschwelliges medizinisches Angebot machen. Sie können ja durchaus noch ein wenig warten.

Und sollten sie doch direkt gleich aus eigenen Stücken in den Kliniken erscheinen, haben alle Häuser heute festgelegte Bereiche für die Erstversorgung der Leichtverletzten, möglichst weit weg von den zentralen Versorgungseinrichtungen.

Die DGU behauptet, so eine einzige Übung würde eine Klinik an die 100000 Euro kosten, weil die an dem Tag ihren Normalbetrieb einstellen muss, auch weil alle OPs dicht sind. 
Sehen wir nicht ganz so. Eine Übung dauert zwischen 3 und 5 Stunden. In dieser Zeit sind keine CTs und keine Röntgenaufnahmen in einer Klinik möglich. Operationen muss bei uns hingegen keine Klinik verschieben. Das wird lediglich simuliert. Nach unseren Kalkulationen hat eine Klinik aber in der Tat je nach Größe 40000 bis 120000 Euro an Ausfallkosten für eine einzige Übung. Und das muss natürlich finanziert werden.

Ja bitte, von wem?

Das ist bekanntlich eine schwierige Diskussion. Das Land Hessen hat schon in den Genehmigungsbescheiden für die Zulassung die Krankenhäuser verpflichtet, bei besonderen Lagen diese Leistungen zu erbringen, gibt dafür aber kein Geld extra. Ein Klinikum kann die zusätzlich entstehenden Kosten andererseits auch nicht durch die Krankenkassen erlösen, es bleibt darauf sitzen. Die Kassen sagen, Katastrophenmedizin ist Daseinsvorsorge und die fällt nicht in unseren Versichertenauftrag. Ich finde aber, die Kosten müssten von den Sozialversicherungsträgern im Sinne einer ganzheitlichen Versorgung getragen werden.

Dafür gibt es die Forderung, dass der Rettungsdienst in das SGB V als Teil der Leistungen der Versicherungen aufgenommen werden sollte.

Ja, und die Forderung mache auch ich mir zu eigen. Das könnte auch die Chancen auf Verbesserungen erhöhen. Ohne Zweifel haben wir zu viele Player in dem Ganzen. Zu viele Berufene und fehlende Standards.

\section{Ein Beispiel?}

Wir diskutieren gerade Konzepte, ob wir in Kliniken Depots für Rettungsdienste einrichten können. Wenn heute ein Wagen mit dem Patienten kommt, ist es in der Regel so, dass der Rettungsdienst sein ganzes Gerät wieder mitnimmt, sobald er den Patienten übergibt. Nun wissen wir aus der
Traumaversorgung, dass es nach medizinischen Kriterien nicht gut ist, wenn man das ganze Equipment, das am Patienten ist, mal so eben abbauen und tauschen muss. Wir würden daher gerne in Kliniken zentrale Depots anlegen, in denen sich der Rettungsdienst bedient, sodass der Materialtausch am Patienten entfallen kann.

\section{Der Dienst nimmt sich ein frisches Set} mit?

Ja, das geht aber derzeit nicht, da wir allein um Frankfurt 5 Rettungsdienstbereiche haben, verschiedene Gebietskörperschaften, in denen auch unterschiedliche Dienste unterwegs sind. Und überall ist das Equipment ein bisschen anders. Diese sehr feingliedrige Aufteilung, da müsste ein Standard her.

Weitere Infos zu Ivena:

http://www.ivena.de

Das Interview führte Bernhard Epping. 